\title{
10006 A Novel Technique of Femoral Artery Technical Angiography for the Detection of Post-bifurcation Puncture and the Avoidance of Complication
}

Satoshi Koizumi, Masaaki Shojima, Shogo Dofuku, and Nobuhito Saito

Objective: Cerebral diagnostic angiography and endovascular surgery are commonly performed via the femoral artery, which is prone to complications. The objective of this study is to describe our approach to achieve femoral access safely. Case Presentations: Between April 2014 and March 2015, we performed femoral angiography from the outer sheath of the intra-arterial cannula prior to the insertion of the sheath. Using those images, we retrospectively divided the puncture status of each patient into four categories: optimal, high-position, low-position, and post-bifurcation punctures.

Of the 190 femoral punctures performed during the study period, femoral arteriograms were confirmed in $155(81.6 \%)$ cases. In those cases, optimal, high-position, low-position, and post-bifurcation punctures accounted for 125 (80.6\%), 1, 0, and 29 cases, respectively. In other words, post-bifurcation puncture could be overlooked in as many as 29/154 (18.8\%) cases without femoral angiography. In the cases of post-bifurcation punctures, large sheath insertion was avoided. Overall, there were no cases of morbidity or mortality related to the puncture.

Conclusion: Femoral artery angiography can visualize post-bifurcation punctures, which are not evident upon the usual fluoroscopy-guided femoral punctures. This method represents a safe and effective way to reduce the risk of complications associated with femoral artery punctures in cerebrovascular catheter angiography and neuroendovascular treatments.

Keywords > endovascular surgery, complication, puncture, femoral, anatomy

\section{Introduction}

Cerebral diagnostic angiography and endovascular surgery are commonly performed via the femoral artery, owing largely to the device profiles and the anatomical accessibility. However, in cardiac interventions, this approach has been reported to be associated with increased risks of complications compared to the radial approach. ${ }^{1)}$ In a meta-analysis of non-cardiac interventions, the complication rate of procedures involving femoral access was reported to range between $3.1 \%-11.4 \% .^{2)}$ To reduce this complication risk, we perform

Department of Neurosurgery, The University of Tokyo, Tokyo, Japan

Received: January 19, 2016; Accepted: March 31, 2016 Corresponding author: Satoshi Koizumi. Department of Neurosurgery, The University of Tokyo Hospital, 7-3-1, Hongo, Bunkyo-ku, Tokyo 113-8655, Japan

Email: sakoizumi-tky@umin.net

(C)2016 The Editorial Committee of Journal of Neuroendovascular Therapy. All rights reserved. femoral artery angiography at our institution. Herein, we describe our specific technique for femoral artery angiography and report on its safety and effectiveness.

\section{Case Presentation}

\section{Method}

Based on previous reports, we defined the optimal puncture site for the femoral artery as the common femoral artery below the inguinal ligament and over the lower edge of the femoral head (Fig. 1). Punctures above the inguinal ligament were defined as high-position punctures, and these are associated with an increased risk of retroperitoneal hemorrhage. ${ }^{3)}$ In angiography, the ligament itself is invisible, but the lowest point of the inferior epigastric artery in its sweep provides the best estimate for the path taken by the ligament. ${ }^{4)}$ Punctures below the femoral head, defined as low-position punctures, are predisposed to hematomas, pseudoaneurysms, and arteriovenous fistulas, owing to incomplete compression. ${ }^{5)}$ Further, even if the puncture seems optimal in relation 


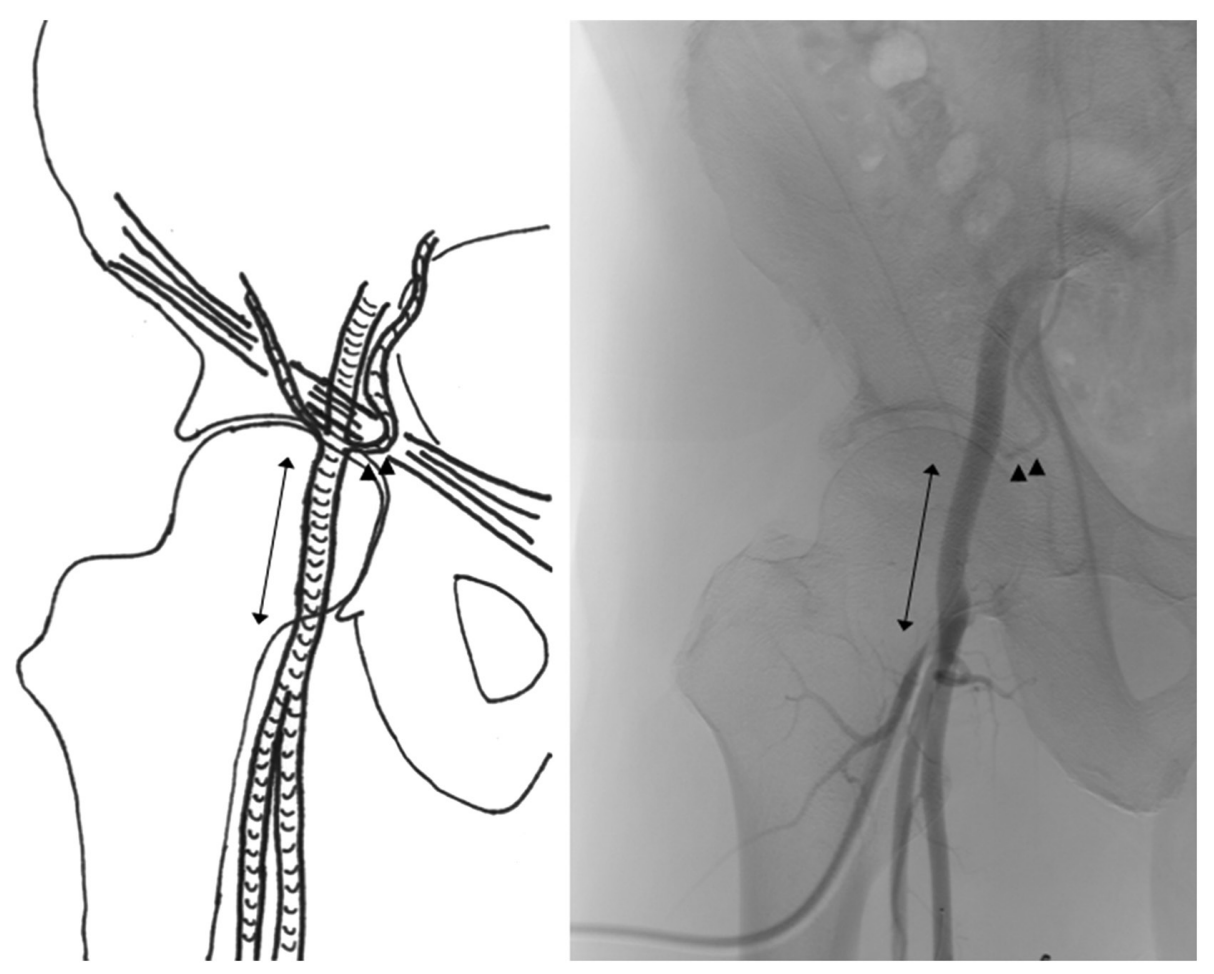

Fig. 1 Schematic (left) and fluoroscopic (right) images of the femoral artery in relation to the surrounding structures. Note that the inguinal ligament is not visible on the fluoroscopic image. Instead, the lowest point of the inferior epigastric artery in its sweep (black arrowheads) provides the best estimate for the path taken by the ligament. The black arrows indicate the range of the optimal puncture point, the common femoral artery below the inguinal ligament and over the lower edge of the femoral head.

to the level of the femoral head, there remains a possibility of post-bifurcation puncture: the puncture of either the superficial or deep femoral artery. Post-bifurcation puncture can lead to vessel obstruction, especially in cases of large-diameter sheath insertion or hemostatic device usage. ${ }^{6}$ In this study, we defined post-bifurcation puncture as the puncture below the common femoral artery in spite that level of the puncture is above the lower edge of the femoral head.

To assess the puncture point and avoid inappropriate puncture, we perform femoral angiography before the insertion of the sheath. Before the puncture, we confirm the position of the femoral head using fluoroscopy, with the aim that the puncture point to the artery would be at the level of the center of the femoral head. Femoral puncture is performed with the usual cannula and a 0.035 -inch $(0.889-\mathrm{mm})$ guidewire. Once the backflow of blood from the artery to the cannula is confirmed, the guidewire is inserted into the artery through the outer sheath of the cannula, and this outer sheath is then pushed forward to the arterial cavity through the guidewire. Subsequently, a contrast media-filled $10 \mathrm{~mL}$-syringe is attached to the outer sheath of the cannula, either with or without an extension tube, and an arteriogram of the femoral artery is obtained by manual injection. After accumulating a number of cases, arteriography with the extension tube became the preferred approach in order to avoid irradiation of the physician's hand. After the arteriogram, the connected tube and syringe are all removed and the guidewire is reinserted to the artery. Through this wire, an arterial sheath is inserted using Seldinger's technique. ${ }^{\text {) }}$ This femoral artery angiography method was not performed in cases of, for example, chronic renal failure or in patients with unstable vital signs.

\section{Results}

Using these definitions of appropriate puncture and the femoral arteriogram technique, we retrospectively reviewed the outcomes and complications of catheter angiography and endovascular surgery performed at our institute. During April 2014 to March 2015, 229 punctures were performed at our institute during 217 sessions of catheter angiography (163 punctures during 156 sessions) and endovascular surgery (66 punctures during 61 sessions), considering bilateral punctures at 1 session. Among them, 190 punctures were performed via the femoral approach. The puncture site confirmation using the femoral arteriography described 

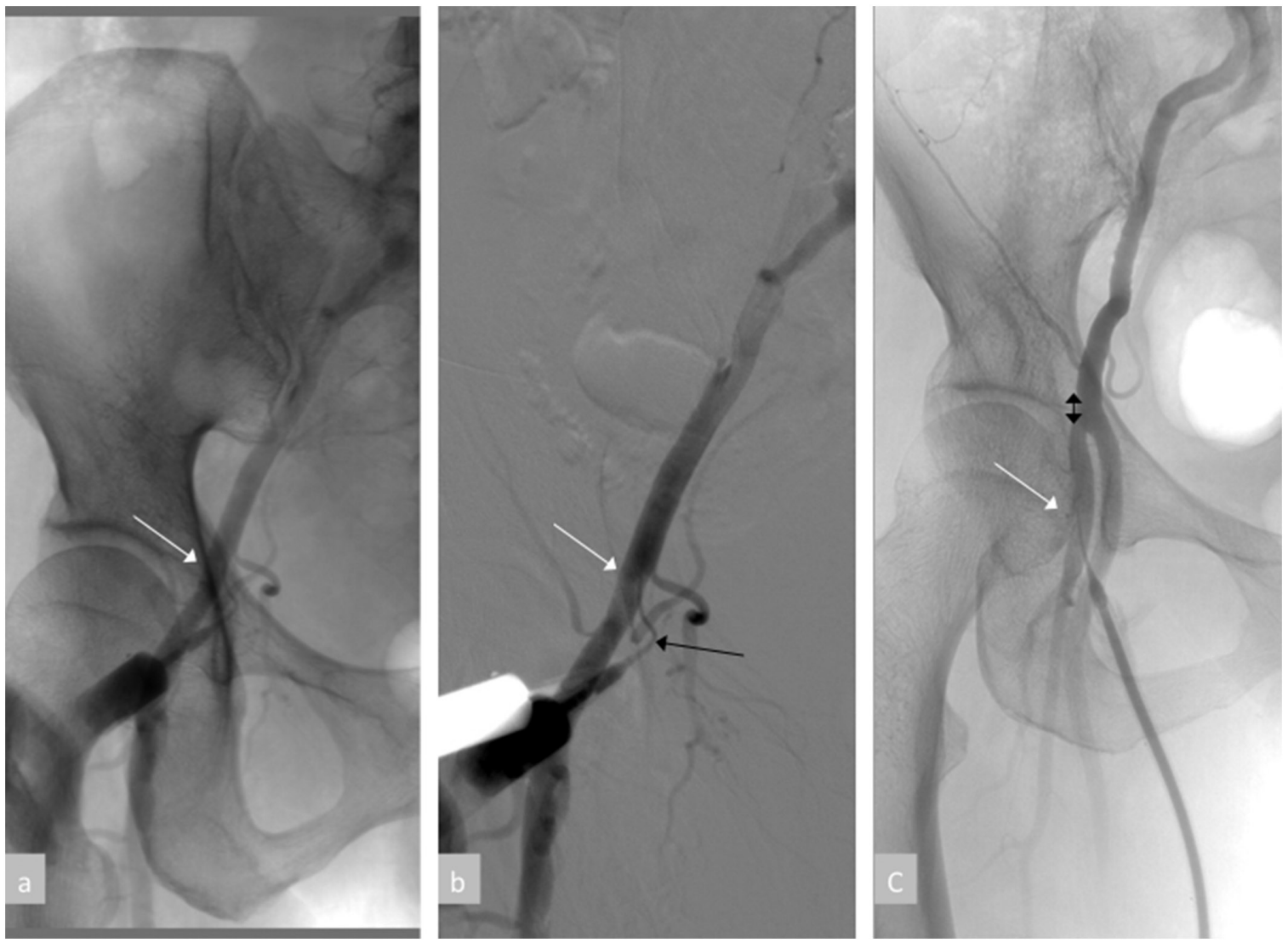

Fig. 2 Examples of inappropriate punctures. Raw (a) and subtracted (b) images of a high-position puncture. Note that the inferior steep of the inferior epigastric artery (black arrow in (b) is below the puncture point, indicating that the puncture is located above the inguinal ligament. High-position punctures are prone to retroperitoneal hemorrhage. (c) An example of postbifurcation puncture. In this patient, access to the common femoral artery is practically hard (the range of optimal puncture point is shown by the black arrow). Post-bifurcation punctures are not suitable for large sheath insertion or hemostatic device usage. In both cases, the puncture points (white arrows) seem normal in relation to the femoral head, implying that fluoroscopy-guided puncture is not enough to discriminate inappropriate punctures.

above was performed in 155 cases $(81.6 \%)$. There were no complications related to the femoral angiography.

Among those 155 punctures, optimal puncture was accomplished in 125 cases (80.6\%). Among the remaining 30 cases, there were 1, 0 , and 29 high-position, low-position, and post-bifurcation punctures, respectively (examples of those inappropriate punctures are shown in Fig. 2). In the case of high-position puncture, the cannula was removed and the puncture site was manually compressed. After hemostasis was confirmed, the femoral puncture was re-performed from the ipsilateral femoral artery. In the cases of post-bifurcation puncture, usual 4-Fr sheath insertion was performed and hemostasis was accomplished by manual compression. If larger sheath insertion was needed, the post-bifurcation puncture side was abandoned and the contralateral femoral artery was selected for the sheath insertion. In the contralateral femoral puncture, femoral artery angiography from the cannula outer sheath was again performed. During the study period, all of those contralateral punctures were optimal.
Our standard strategy of femoral artery puncture is summarized in Fig. 3. Among all 190 femoral punctures, there was only one complication, namely re-bleeding after the postoperative ambulation. In this case, optimal puncture was confirmed by femoral angiography and a hemostatic device (Angioseal $^{\mathrm{TM}}$, St. Jude Medical Inc., St. Paul, MN, USA) was used. After re-bleeding, manual compression was performed immediately and the patient did not suffer any continuing morbidity. Hence, there was no case of morbidity or mortality related to the puncture during the study period.

\section{Discussion}

As shown in our retrospective review, femoral artery angiography following fluoroscopy-guided femoral puncture is a relatively safe and simple technique to rule out inappropriate punctures. In previous studies, fluoroscopy-guided puncture confirming the femoral head has been shown to be effective in avoiding puncture site complications. ${ }^{8,9)}$ However, 


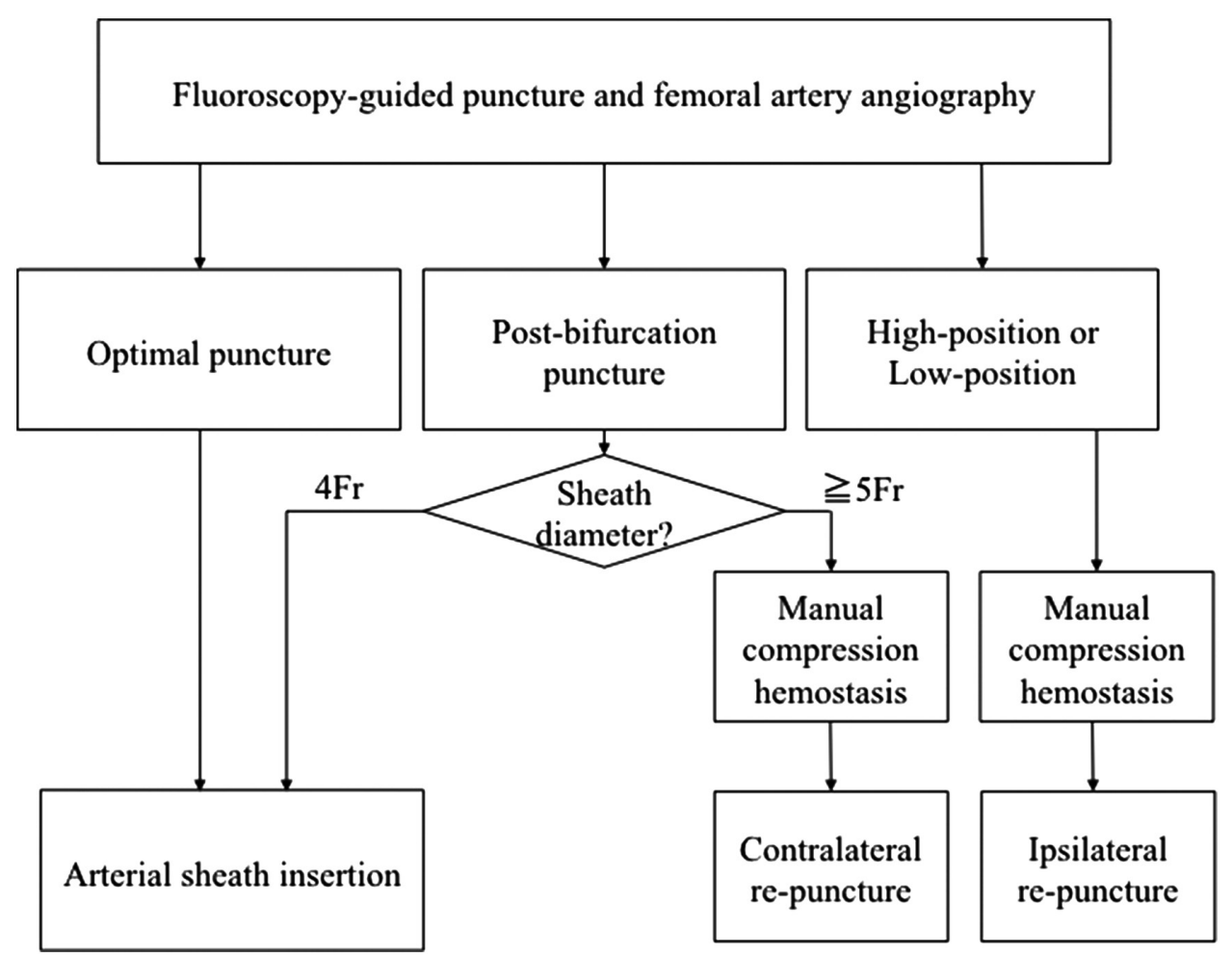

Fig. 3 Our strategy of femoral artery puncture using the femoral angiography technique. In cases of post-puncture bifurcation, large sheath insertion is avoided and contralateral re-puncture is attempted. In cases of high or low-position punctures, manual compression hemostasis followed by ipsilateral re-puncture is the solution. In practice, however, high or low punctures rarely occur under fluoroscopic guidance.

fluoroscopy-guided punctures cannot avoid post-bifurcation punctures, which occurred in $18.8 \%(29 / 154)$ of our cases. In the literature, high bifurcation of the common femoral artery; that is, above the inferior border of the femoral head, is reported to account for as many as $30 \%$ of the patients. ${ }^{10)}$

There is a consensus that the larger the sheath-to-artery size ratio, the greater the risk of vascular obstruction and closure failure. The superficial femoral artery and deep femoral artery both taper as they branch from the common femoral artery. ${ }^{11)}$ In cases of post-bifurcation punctures the risk of late vessel stenosis or occlusion can become higher especially if hemostatic devices, such as Angioseal ${ }^{\mathrm{TM}}$, are used. ${ }^{12)}$ Therefore, the confirmation of the femoral bifurcation level is recommended. To achieve this, angiogram via a 4-Fr micropuncture sheath has also been reported. ${ }^{11)}$ However, our technique is considered superior in the point that we can obtain the angiogram before the insertion of the arterial sheath; that is, the diameter of the arterial wall puncture hole is only the size of cannula at the time of femoral angiography. As a result, manual compression hemostasis and re-puncture, if needed, become much easier than the 4-Fr sheath insertion method.
Our study has some limitations. First, our data were retrospective and obtained from a single center. More centers and studies should focus on identifying the appropriate way to perform optimal punctures. Second, our technique cannot recognize post-bifurcation punctures before the puncture of the arterial wall itself. To avoid post-puncture bifurcation, ultrasound guidance has been reported to be effective. ${ }^{13,14)}$ However, in our study, recognition of post-bifurcation punctures after the cannula insertion did not have any effect on the rate of complications. Hence, from the viewpoint of convenience, we believe that our angiography technique is sufficiently effective for reducing the complication rate. Ultrasound-guided puncture can be considered for especially high-risk patients, such as those $>75$ years old, those with congestive heart failure, and those taking anticoagulants. ${ }^{14)}$ Moreover, our strategy does not answer how to deal with the bilateral high-bifurcation cases. ${ }^{10)}$ In our series, there was no bilateral post-bifurcation puncture. However, more attention should be paid if the high-bifurcation puncture is confirmed on one side and the contralateral puncture is attempted. Contralateral femoral artery angiography through the arterial sheath can be a solution to achieve optimal contralateral 
puncture, provided that the risk of bilateral sheath insertion permitted. Alternatively, ultrasound-guided contralateral puncture may serve as a useful option.

In Japan, the puncture site complication rate in neuroendovascular treatment has been reported to be relatively low $(0.63 \%)$ in JR-NET $\left.1 / 2 .{ }^{15}\right)$ Nevertheless, the importance of avoiding puncture site complications cannot be exaggerated enough. We believe that our femoral artery angiography technique may represent a useful tool to perform safer femoral punctures.

\section{Conclusion}

Femoral artery angiography can visualize post-bifurcation punctures, which are not evident upon usual fluoroscopyguided femoral punctures. It is a safe and effective way to reduce the complication rate of femoral artery punctures in cerebrovascular catheter angiography and neuroendovascular treatments.

\section{Disclosure Statement}

The authors declare that they have no conflict of interest.

\section{References}

1) Jang JS, Jin HY, Seo JS, et al: The transradial versus the transfemoral approach for primary percutaneous coronary intervention in patients with acute myocardial infarction: a systematic review and meta-analysis. EuroIntervention 2012; 8: 501-510.

2) Das R, Ahmed K, Athanasiou T, et al: Arterial closure devices versus manual compression for femoral haemostasis in interventional radiological procedures: a systematic review and meta-analysis. Cardiovasc Intervent Radiol 2011; 34: 723-738.

3) Ellis SG, Bhatt D, Kapadia S, et al: Correlates and outcomes of retroperitoneal hemorrhage complicating percutaneous coronary intervention. Catheter Cardiovasc Interv 2006; 67: $541-545$.

4) Turi ZG: An evidence-based approach to femoral arterial access and closure. Rev Cardiovasc Med 2008; 9: 7-18.
5) Turi ZG: Optimal femoral access prevents complications. Cardiac Interventions Today 2008; 35-38.

6) Ahn HY, Lee HJ, Lee HJ, et al: Assessment of the optimal site of femoral artery puncture and angiographic anatomical study of the common femoral artery. J Korean Neurosurg Soc 2014; 56: 91-97.

7) Seldinger SI: Catheter replacement of the needle in percutaneous arteriography; a new technique. Acta radiol 1953; 39: 368-376.

8) Abu-Fadel MS, Sparling JM, Zacharias SJ, et al: Fluoroscopy vs. traditional guided femoral arterial access and the use of closure devices: a randomized controlled trial. Catheter Cardiovasc Interv 2009; 74: 533-539.

9) Fitts J, Ver Lee P, Hofmaster P, et al: Fluoroscopy-guided femoral artery puncture reduces the risk of PCI-related vascular complications. J Interv Cardiol 2008; 21: 273-278.

10) Gupta V, Feng K, Cheruvu P, et al: High femoral artery bifurcation predicts contralateral high bifurcation: implications for complex percutaneous cardiovascular procedures requiring large caliber and/or dual access. J Invasive Cardiol 2014; 26 : 409-412.

11) Cilingiroglu $M$, Feldman $T$, Salinger $M H$, et al: Fluoroscopically-guided micropuncture femoral artery access for large-caliber sheath insertion. J Invasive Cardiol 2011; 23: $157-161$.

12) Hussain T, Al-Hamali S: Femoral artery occlusion with a percutaneous arterial closure device after a routine coronary angiogram: a case report and literature review. Ann R Coll Surg Engl 2011; 93: e102-e104.

13) Seto AH, Abu-Fadel MS, Sparling JM, et al: Real-time ultrasound guidance facilitates femoral arterial access and reduces vascular complications: FAUST (Femoral Arterial Access With Ultrasound Trial). JACC Cardiovasc Interv 2010; 3: 751-758.

14) Lo RC, Fokkema MT, Curran $T$, et al: Routine use of ultrasound-guided access reduces access site-related complications after lower extremity percutaneous revascularization. J Vasc Surg 2015; 61: 405-12.

15) Sato M, Matsumaru Y, Sakai N, et al: Detailed analysis of puncture site vascular complications in Japanese Registry of Neuroendovascular Therapy (JR-NET) and JR-NET2. Neurol Med Chir (Tokyo) 2014; 54: 17-22. 